\title{
Erratum to: Smoking withdrawal is associated with increases in brain activation during decision making and reward anticipation: A preliminary study
}

\author{
Merideth A. Addicott • David A. A. Baranger • \\ Rachel V. Kozink • Moria J. Smoski • \\ Gabriel S. Dichter • F. Joseph McClernon
}

Received: 30 September 2011 / Accepted: 30 September 2011 /Published online: 18 October 2011

(C) Springer-Verlag 2011

\section{Erratum to: Psychopharmacology \\ DOI 10.1007/s00213-011-2404-3}

Due to a conversion error, the number of voxels necessary for cluster correction was miscalculated and should have been $k=313$ instead of $k=53$ voxels as reported. We have updated the results table (see below) to now indicate which clusters remain significant at the correct cluster-corrected threshold $(p<.01 ; k=313$; cluster-corrected to $p<.05)$ and which are significant at uncorrected thresholds of $p<.001$, $k=10$ and $p<.005, k=10$. The only previously reported clusters not significant at any of these thresholds are bilateral paracingulate cortices during the Selection Phase only. References to bilateral paracingulate activation during the selection phase in the Results, Discussion and Figure 3 can be disregarded.

An updated interpretation of our results, focused primarily on activations that survived cluster correction, suggests that smoking abstinence increases BOLD response during the selection phase of the WoF task in somatosensory (post central gyrus, rolandic operculum), visual (lingual gyrus), and insular cortices. Abstinence also increases BOLD response during the anticipation phase of the task in right frontal pole, potentially reflecting the monitoring of reward values during decision making.

While other activations do not survive cluster correction, their inclusion, particularly in the context of a preliminary/exploratory study, still add meaningful infor-

The online version of the original article can be found at http://dx.doi. org/10.1007/s00213-011-2404-3.

M. A. Addicott • D. A. A. Baranger - R. V. Kozink •

M. J. Smoski · G. S. Dichter • F. J. McClernon $(\triangle)$

Department of Psychiatry and Behavioral Sciences,

Duke University Medical Center,

Box 2701, Durham, NC 27708, USA

e-mail: mccle011@mc.duke.edu

M. A. Addicott $\cdot$ D. A. A. Baranger $\cdot$ R. V. Kozink

M. J. Smoski · G. S. Dichter · F. J. McClernon

Duke-UNC Brain Imaging and Analysis Center,

Durham, NC, USA

G. S. Dichter

Department of Psychiatry, School of Medicine,

University of North Carolina at Chapel Hill,

Chapel Hill, NC, USA

\section{F. J. McClernon}

Durham Veterans Affairs Medical Center, VISN 6 Mental Illness,

Research, Education, and Clinical Center,

Durham, NC, USA 
Table 2 Areas showing significant activation during the Wheel of Fortune task adenotes area of overlapping activation in post hoc analysis with RT covariate.

$p<.01$ and cluster-corrected to $p<.05$, unless otherwise indicated.

${ }^{\dagger} p<.001, k=10$

$\ddagger p<.005, k=10$

\begin{tabular}{|c|c|c|c|c|c|c|}
\hline \multirow[b]{2}{*}{ Brain area } & \multirow[b]{2}{*}{ Side } & \multirow[b]{2}{*}{$\#$ of voxels } & \multirow[b]{2}{*}{$\mathrm{Z} \max$} & \multicolumn{3}{|c|}{ MNI Coordinates } \\
\hline & & & & $\mathrm{x}$ & $\mathrm{y}$ & $\mathrm{Z}$ \\
\hline \multicolumn{7}{|c|}{ Selection phase (Abstinent $>$ Satiated, Money $>$ Control) } \\
\hline Postcentral Gyrus & $\mathrm{L}$ & 1072 & 3.28 & -26 & -32 & 60 \\
\hline Insula & $\mathrm{R}$ & 393 & 3.43 & 44 & -8 & -12 \\
\hline Lingual Gyrus ${ }^{\text {a }}$ & $\mathrm{L}$ & 385 & 2.80 & -16 & -56 & -4 \\
\hline Rolandic Operculum ${ }^{a}$ & $\mathrm{R}$ & 362 & 3.61 & 52 & -26 & 22 \\
\hline Postcentral Gyrus & $\mathrm{R}$ & 333 & 2.97 & 34 & -30 & 54 \\
\hline Cerebellum ${ }^{\ddagger}$ & $\mathrm{L}$ & 256 & 3.05 & -2 & -54 & -16 \\
\hline Rolandic Operculum \$,a & $\mathrm{L}$ & 241 & 3.07 & -60 & 4 & 4 \\
\hline Insula & $\mathrm{R}$ & 227 & 2.88 & 36 & -8 & -8 \\
\hline Middle Frontal Gyrus $\stackrel{\ddagger}{\ddagger}$ & $\mathrm{L}$ & 183 & 3.10 & -34 & 52 & 8 \\
\hline Precentral Gyrus ${ }^{*}$ & $\mathrm{R}$ & 164 & 2.67 & 34 & -8 & 46 \\
\hline Middle Occipital Gyrus & $\mathrm{L}$ & 163 & 3.04 & -40 & -70 & 6 \\
\hline Supplementary Motor Area & $\mathrm{R}$ & 159 & 3.05 & 14 & -2 & 68 \\
\hline Supracalcarine Cortex ${ }^{\ddagger}, \mathrm{a}$ & $\mathrm{R}$ & 155 & 3.08 & 22 & -66 & 16 \\
\hline Middle Frontal Gyrus ${ }^{*}$ & $\mathrm{~L}$ & 144 & 3.07 & -30 & 22 & 58 \\
\hline Inferior Frontal Gyrus $\$$,a & $\mathrm{R}$ & 131 & 3.19 & 52 & 36 & -14 \\
\hline Precuneus $\$$,a & $\mathrm{L}$ & 124 & 2.95 & -2 & -60 & 12 \\
\hline Orbital Frontal Gyrus $\$$,a & $\mathrm{R}$ & 55 & 2.89 & 4 & 54 & -24 \\
\hline \multicolumn{7}{|c|}{ Anticipation phase (Abstinent $>$ Satiated, Money $>$ Control) } \\
\hline Frontal Pole & $\mathrm{R}$ & 336 & 3.46 & 26 & 46 & 20 \\
\hline Insula ${ }^{\dagger}$ & $\mathrm{L}$ & 255 & 3.36 & -38 & 6 & -2 \\
\hline Paracingulate Cortex & $\mathrm{R}$ & 139 & 2.77 & 14 & 40 & -6 \\
\hline \multicolumn{7}{|c|}{ Anticipation phase (Satiated $>$ Abstinent, Money $>$ Control) } \\
\hline Precentral Gyrus & $\mathrm{L}$ & 394 & 2.8 & -2 & -28 & 56 \\
\hline Putamen ${ }^{\ddagger}$ & $\mathrm{R}$ & 53 & 3.05 & 28 & -14 & -2 \\
\hline \multicolumn{7}{|c|}{ Outcome phase (Satiated $>$ Abstinent, Win $>$ No Win) } \\
\hline Paracingulate Cortex & $\mathrm{L}$ & 122 & 3.04 & -2 & 48 & -6 \\
\hline
\end{tabular}

mation to the literature. Many of the regions in which smoking condition differences were observed at the uncorrected thresholds are regions which have been previously implicated in decision making and in research using the
Wheel of Fortune task (e.g., precuneus, insula, putamen). We regret any confusion associated with this correction and, as with all preliminary studies, look forward to future replication of these findings. 